\title{
El gran pago de Mulsina o el arte de mover las montañas
}

Le grand rituel d'offrandes à Mulsina ou l'art de déplacer les montagnes The great ritual at Mulsina or the art of moving mountains

\section{Xavier Bellenger}

\section{(2) OpenEdition}

Journals

Edición electrónica

URL: http://journals.openedition.org/bifea/5506

DOI: $10.4000 /$ bifea.5506

ISSN: 2076-5827

Editor

Institut Français d'Études Andines

Edición impresa

Fecha de publicación: 1 agosto 2005

Paginación: 235-249

ISSN: 0303-7495

\section{Referencia electrónica}

Xavier Bellenger, «El gran pago de Mulsina o el arte de mover las montañas », Bulletin de l'Institut français d'études andines [En línea], 34 (2) | 2005, Publicado el 08 agosto 2005, consultado el 10

diciembre 2020. URL : http://journals.openedition.org/bifea/5506 ; DOI : https://doi.org/10.4000/bifea 5506

\section{(c) $(1)$}

Les contenus du Bulletin de l'Institut français d'études andines sont mis à disposition selon les termes de la licence Creative Commons Attribution - Pas d'Utilisation Commerciale - Pas de Modification 4.0 International. 


\title{
El gran pago de Mulsina o el arte de mover las montañas
}

\author{
Xavier Bellenger*
}

\begin{abstract}
Resumen
La aprehensión de la geografía, tal como es enfocada durante las prácticas rituales profilácticas realizadas en Taquile, pequeña isla peruana del lago Titicaca, aporta importantes datos sobre la disposición y la percepción del espacio sagrado por parte de los miembros de esta comunidad insular agraria de lengua quechua. Igualmente, el modo operatorio practicado por los paqu o chamanes de la isla para granjearse los favores de las divinidades circumlacustres revela estrategias ancestrales, desplegadas durante complejos rituales, destinados a atraer hacia sí, aysamu-1 ${ }^{1}$, y a hacer mover fuerzas tutelares exteriores a la isla de Taquile. Estas, a veces distantes unas de otras por varias centenas de kilómetros, son invitadas una vez al año a Mulsina, en la cima de la isla, a un banquete en donde el análisis de las cenizas de los alimentos consumidos predecirá el devenir de la comunidad.
\end{abstract}

Palabras clave - Andes, Apu, culto de los ancestros, ciclo agrario, divinidades, espacio sagrado, cerros, isla de Taquile, lago Titicaca, Perú, santuarios, ritual, ofrendas, geografía, chamanes, pachamama, coca

\section{Le grand rituel d'offrandes à Mulsina ou l'art de déplacer les montagnes}

\section{Résumé}

L'appréhension de la géographie, telle qu'elle est envisagée au cours de pratiques rituelles prophylactiques réalisées à Taquile, petite île péruvienne du lac Titicaca, livre d'importantes données sur l'agencement et la perception de l'espace sacré par les membres de cette communauté insulaire agraire de langue quechua. De même, le mode opératoire pratiqué par les paqu ou chamans de

* IFEA, Casilla 18-1217, Lima 18 Perú. Correos electrónicos: xbellenger@compuserve.com, bxandino@hotmail.com

1 Aysamu-, término quechua construido a partir de la raíz verbal aysa- que significa: «jalado por una rienda o una asa» (Itier, 1997: 159) y del sufijo -mu- que agregado a los verbos de movimiento, indica que la acción comienza en un lugar más o menos alejado y se dirige hacia el locutor (Cusihuamán, 2001: 201-202). 
l'île pour s'attirer les bienfaits de divinités circumlacustres, révèle d'ancestrales stratégies, déployées au cours de rituels complexes, destinés à attirer à soi, et à faire se déplacer des puissances tutélaires extérieures à l'île de Taquile. Celles-ci, parfois distantes les unes des autres de plusieurs centaines de kilomètres, sont conviées une fois l'an à Mulsina, au sommet de l'île, à un banquet dont l'analyse des cendres des mets consumés servira à prédire le devenir de la communauté.

Mots clés - Andes, Apu, culte des ancêtres, cycle agraire, divinités, espace sacré, montagnes, île de Taquile, lac Titicaca, Pérou, santuaire, rituel, offrandes, géographie, chamane, pachamama, coca

\title{
The great ritual at Mulsina or the art of moving mountains
}

\begin{abstract}
The local geography as it is perceived during protective rituals carried out on Taquile, a small island in Lake Titicaca, provides important data about the organization and perception of a sacred area as it is viewed by members of this insular and agrarian Quechua community. Complex rituals practiced by shamans of the island in order to attract the favourable influence of the divinities (aysamuy) bordering the lake, reveal ancestral strategies. The practices aim at attracting the divinities unto the shamans themselves and to influence tutelary powers located outside the island. The latter divinities separated from one another by hundreds of kilometers are invited once a year to a banquet held at the sanctuary of Mulsina, on the summit of the island. At this occasion, an interpretation of the ashes left over from incinerated offerings contribute to the prediction of the community's future.
\end{abstract}

Key words - Andes, Apu, cult of the ancestors, agrarian cycle, divinities, sacred area, mountains, island of Taquile, Lake Titicaca, Peru, sanctuary, ritual, offerings, geography, shaman, pachamama, coca

Para abordar en este artículo el tema de la geografía sagrada, y más particularmente el del espacio, tantas veces visitado por los antropólogos y por los investigadores de los Andes, hemos escogido en este caso, como punto de vista, aquél vigente en la isla de Taquile, donde residimos de 1999 a 2001, en el marco de un estudio más vasto dedicado a los ciclos rituales y musicales en los Andes².

\section{LA ISLA DE TAQUILE}

Con una población de cerca de 1500 habitantes, la pequeña isla de Taquile emerge de las aguas profundas del lago Titicaca a la salida de la bahía de Puno. A pesar de sus modestas dimensiones

2 Estas investigaciones fueron realizadas como investigador del Instituto Francés de Estudios Andinos. Agradezco encarecidamente a esta institución que ofrece condiciones de investigación excepcionales. 


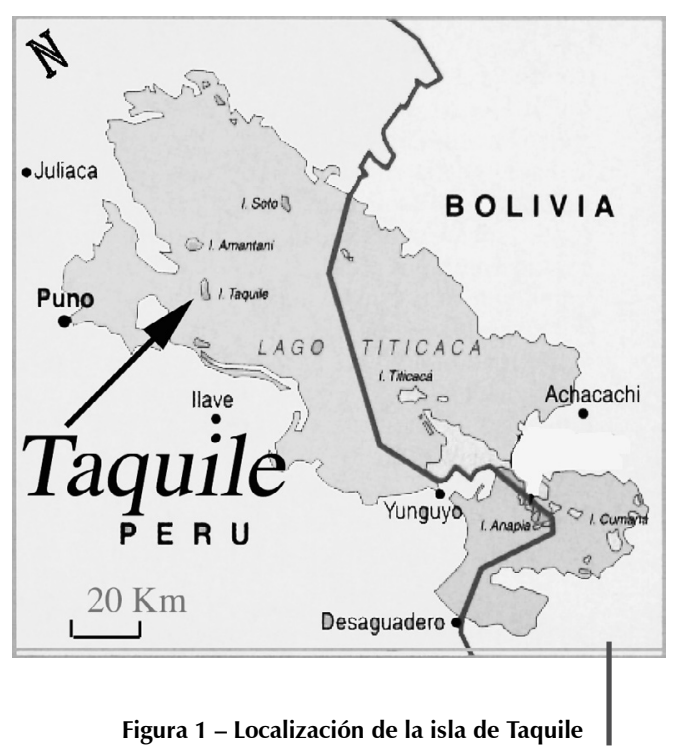

$(6,1 \mathrm{~km}$ en su mayor longitud norte-sur y $1,83 \mathrm{~km}$ de este a oeste) presenta un relieve contrastado. En su punto culminante, situado a $4107 \mathrm{~m}$ de altura, se levanta el más importante santuario de Taquile, conocido bajo el nombre de Mulsina, situado a $15^{\circ} 45^{\prime} 466 \mathrm{~S}, 69^{\circ} 41^{\prime} 371 \mathrm{O}$.

La fisonomía de Taquile presenta contrastes visuales muy claros. Partiendo de la cumbre hacia el sur, una línea de cresta de orientación nortesur corta la isla, flanqueada por una vertiente occidental abrupta que cae hacia el lago. Los andenes de cultivo están ingeniosamente colgadas de ella en un desnivel de más de 250 metros. La línea de la cresta atraviesa enseguida el segundo punto culminante de la isla: Kalapata $(4032 \mathrm{~m})$ ubicado a $15^{\circ} 46^{\prime} 279 \mathrm{~S}, 69^{\circ} 41^{\prime} 230 \mathrm{O}$, el mismo que está alineado con otras cumbres como Pukarapata (3990 m) (15² 46'588 S, $\left.69^{\circ} 41^{\prime} 048 \mathrm{O}\right), \quad$ Tulutepata $\quad(3945 \mathrm{~m})$ $\left(15^{\circ} 47^{\prime} 081 \mathrm{~S}, 69^{\circ} 40^{\prime} 923 \mathrm{O}\right)$ y finalmente Kuwanu pata (3 $941 \mathrm{~m}) \quad\left(15^{\circ} 47^{\prime} 400 \mathrm{~S}\right.$, $69^{\circ} 41^{\prime} 913 \mathrm{O}$ ), en el extremo sur de la isla.

Algunas pequeñas depresiones se intercalan entre estos montes, inclinadas en su mayoría hacia el este, abundantemente cultivadas o también sirviendo como pastizales. Fuera de algunos eucaliptos, la vegetación que marca el territorio es más bien baja. Las tonalidades de la isla oscilan entre el ocre durante la estación seca, de mayo a setiembre, y el verdoso, característico de la estación de lluvias, de octubre a marzo.

Pequeños caminos empedrados atraviesan la isla de par en par, uniendo un hábitat disperso de casas de barro y de piedra con techos de paja progresivamente reemplazados por calamina.

Largo tiempo protegidos, en cierta medida, por su insularidad, los Taquileños han conservado una organización social tradicional, prácticas y rituales que acompañan su vida cotidiana y marcan el ciclo de crecimiento de las plantas cultivadas, esencialmente: la papa, la oxálida tuberosa oca, el maíz y las habas.

Anualmente, mediante rotación de cultivos, esas plantas están distribuidas sobre los territorios llamados suyu (Matos Mar, 1951), ampliamente representados en los numerosos textiles tejidos y llevados por los Taquileños (Braunsberger, 1983).

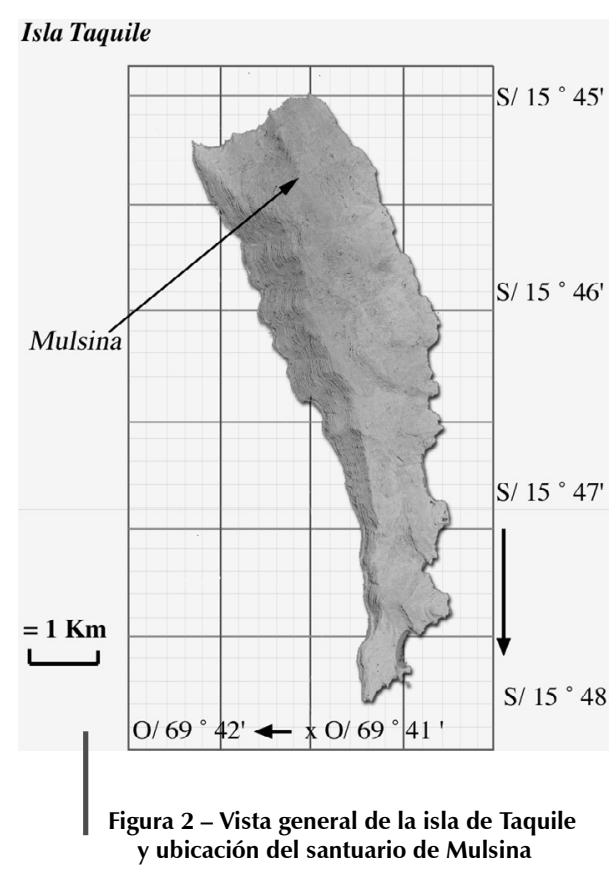

Según la tradición oral de Taquile, la isla habría sido dividida mucho antes de la Conquista en seis territorios o suqta suyu, denominados en nuestra época, de norte a sur: Lakayano, Estancia, Chuñopampa, Kollino, Huayllano y Kollata. Estos territorios están tradicionalmente agrupados en dos conjuntos de una superficie sensiblemente equivalente, situados de un lado y de otro de 

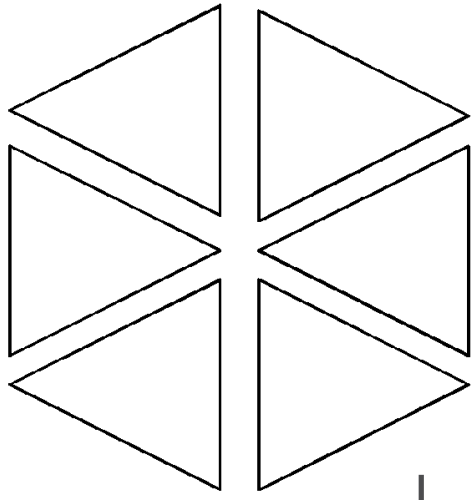

Figura 3 - Símbolo representando los seis suyu o territorios de Taquile

tres suyu y según un orden cíclico inmutable. Se encontrará particularmente esta práctica en diferentes comunidades agrarias quechua o aymara, alrededor del lago, en donde el cultivo de la papa, de la oca, de las habas y del maíz, en forma mixta, se alterna con la puesta en barbecho de las tierras utilizadas generalmente como pastizales.

El conjunto del territorio comunitario de Taquile está bajo la responsabilidad de autoridades tradicionales elegidas todos los años en el mes de noviembre en la plaza pública, levantando la mano. Estas autoridades están constituidas, en su núcleo esencial, por un gobernador o Hatun tayta y cuatro varayuq cuya jurisdicción se aplica para cada uno de ellos a un cuarto del territorio, dos varayuq para la mitad de arriba, dos varayuq para la de abajo. Este modo de administración del espacio y de organización social no es específico de esta isla del lago, se lo encuentra en efecto, bajo formas más o menos desarrolladas en el conjunto de las comunidades tradicionales agrarias andinas.

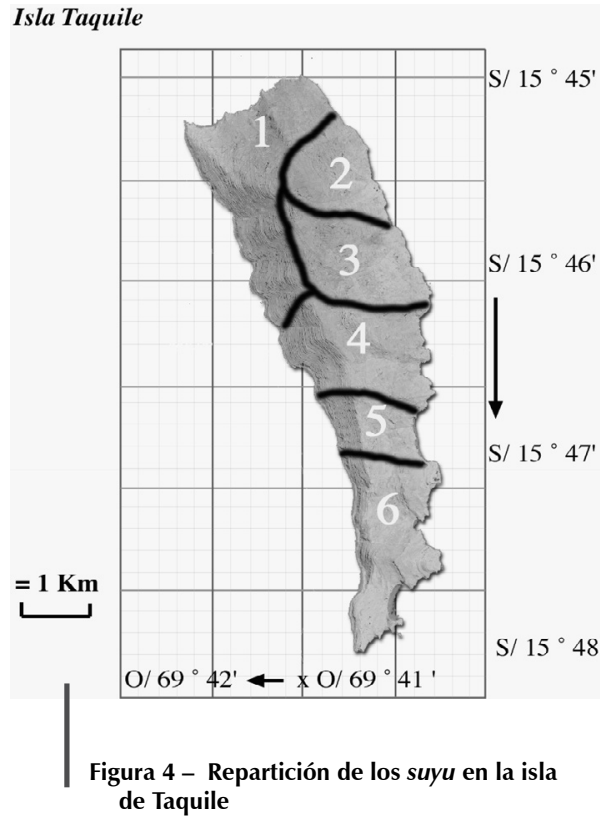

\section{DESTINO DE LAS OFRENDAS ELABORADAS EN EL SANTUARIO DE MULSINA}

Uno de los momentos importantes en el ciclo ritual de la isla de Taquile interviene poco antes del momento crucial de las primeras cosechas, que corresponde a la época de Pascua de Resurección. En este período, las autoridades tradicionales de la isla se dirigen, bajo la conducción de chamanes, al santuario de Mulsina. En este lugar, bajo la conducción de un paqu principal acompañado de asistentes, se prepararán numerosas ofrendas destinadas a la divinidad de la tierra, actualmente invocada con el nombre de pachamama, constituidas esencialmente

3 Mito iskay rumi taquilimanta recopilado en Taquile. Video. Colección Xavier Bellenger. Ref. IFEA XBE-V2-C-00331. TC. (código temporal de inicio) 0h:49:00" (Bellenger, 2001). 
por alimentos considerados como crudos que serán enterrados, y ofrendas calificadas de cocidas e incineradas durante el ritual. Estas últimas están destinadas a los $A p u$, divinidades tutelares o «señores de las montañas» a quienes se implora para que protejan las cosechas y traigan alimento en cantidad. Durante este ritual primordial del Gran pago de Mulsina, el chamán llamará en orden preciso a decenas de lugares situados en Taquile, pero también fuera de la isla, tejiendo, de esta manera, en el espacio líneas mágicas que revelarán poco a poco, durante la ceremonia, una geografía sagrada de la región.

\section{ELEMENTOS PARA NAVEGAR EN EL ESPACIO SAGRADO}

Pero antes de proseguir es necesario adquirir algunos conocimientos de los santuarios de la isla de Taquile y de la región del lago para poder navegar en este espacio sagrado. Estas informaciones esenciales permitirán establecer una relación entre lugares geográficos constantemente invocados durante los rituales, y su emplazamiento simbólico sobre la mesa de ofrendas o mesa del chamán. Los lugares llamados durante la ceremonia, situados en la isla Taquile así como alrededor del lago Titicaca, son considerados santuarios en los que residen las fuerzas tutelares. Las ofrendas ${ }^{4}$ que les están destinadas están constituidas en lo esencial por hojas de coca, generalmente por tres hojas juntas, untadas de grasa de llama llamp'u y humectadas con alcohol. Estas ofrendas llamadas k'intu están dispuestas sobre una hoja de papel, como puntos de un mapa geográfico.

Para establecer la relación entre estos k'intu y los diferentes santuarios a los que están dedicados, hemos tenido que recorrer durante más de seis meses hasta los más pequeños recovecos de la isla de Taquile, así como los lugares situados en todo el contorno del lago Titicaca, y que son invocados durante los rituales. Este aprendizaje de la geografía sagrada ha sido posible gracias a la extrema paciencia de un paqu quien nos ha enseñado además, durante más de dos años, la práctica de los principales rituales.

\section{LA ORGANIZACIÓN ESPACIAL, JERÁRQUICA Y FUNCIONAL DE LOS SANTUARIOS}

Así, durante nuestras investigaciones, hemos registrado y georeferenciado más de una centena de santuarios en Taquile, lo que es bastante para este territorio insular de menos de $12 \mathrm{~km}^{2}$. Estos santuarios aparecen esencialmente bajo la forma de pequeños edificios de piedras superpuestas, de forma cónica, de un tamaño que oscila entre $90 \mathrm{~cm}$, en el caso de los más pequeños, y que puede alcanzar hasta más de $3 \mathrm{~m}$ de alto, en el caso de los más grandes. Generalmente están rematados por una cruz y en nuestros días se los designa con mucha frecuencia bajo el nombre de calvario.

Bajo esta forma han reemplazado con mucha frecuencia a los monolitos, o grandes piedras de formas singulares que señalaban la presencia de antiguas divinidades, que fueron destruidos sistemáticamente desde la época colonial por los españoles y los representantes de la Iglesia Católica.

Durante nuestra estadía, hemos identificado uno a uno estos calvarios y los hemos clasificado por territorios o suyu, según su orden jerárquico y sus poderes específicos.

Algunos de ellos están provistos de un pequeño receptáculo o altar situado en la parte superior del monumento. Este constituye una suerte de abertura que orienta «el ojo» o «la mirada» del calvario hacia diferentes lugares situados esencialmente fuera del territorio insular, generalmente más allá de

4 Para la descripción y el estudio del conjunto de ingredientes que entran en la composición de las ofrendas dedicadas a las divinidades, referirse particularmente a los estudios de Girault (1988) y de Fernández Juárez (1995). 


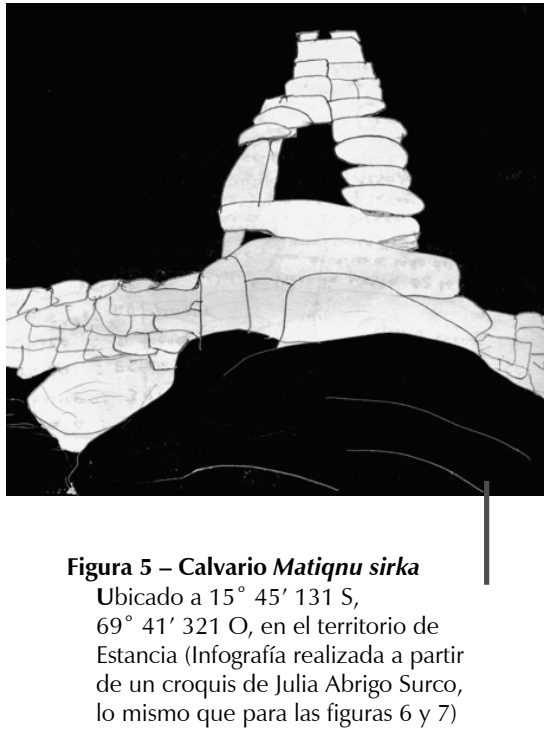

las riberas del lago Titicaca. Salvo escasas excepciones, están orientados hacia el este ${ }^{5}$ y distribuidos de manera desigual sobre el conjunto del territorio de la isla de Taquile.

Cada uno de los seis territorios o suyu de la isla de Taquile está colocado bajo la influencia de una fuerza tutelar llamada Apu mayor, materializada por un túmulo de gran tamaño designado como calvario mayor, que domina el territorio puesto bajo su protección.

A cada uno de estos seis santuarios preeminentes, en su mayoría rodeados de un muro pequeño, está asociado un santuario enterrado, a menudo mantenido en secreto, llamado churu ${ }^{6}$, en cuya proximidad son preparados dos tipos de ofrendas. Por un lado, las ofrendas consideradas como alimento cocido, destinadas a los Apu, esencialmente constituidas de $k^{\prime}$ intu u hojas de coca seleccionadas que serán incineradas en una fogata preparada con este fín, y en donde la interpretación del color de las cenizas residuales revelará la aceptación o el rechazo del sacrificio por parte de las divinidades de las montañas. Por otro lado, con ocasión de los grandes rituales o grandes pagos, están asociados alimentos llamados cocidos con otras ofrendas comunitarias calificadas de crudas, destinadas a la tierra

o Pachamama. Están constituidas en particular por golosinas, así como por mixturas de agua mezclada con maíz contenidas en vasijas o chuwa chatu. Estos alimentos crudos están dispuestos en un receptáculo enterrado que será sellado, el churu, y luego recubierto con piedras con el fin de ocultarlo.

De cada Calvario mayor que domina un territorio o suyu dependen otros dos santuarios de tamaño más pequeño, o calvarios menores, considerados como sus asistentes o ayudantes. Generalmente estos santuarios menores son requeridos por los agricultores durante los cultos de proximidad y son invocados localmente durante ritos familiares. Estos calvarios menores están también asociados con otros pequeños tumulus edificados por los Taquileños en los lugares «visitados por el relámpago», para librarlos del rayo o proteger sus sembrados del granizo.

Los santuarios más importantes de la isla de Taquile, cuyo poder podría ser fatal al común de los mortales, no pueden ser visitados o requeridos por los no iniciados sin la presencia de los paqu. Son particularmente requeridos durante la estación seca para llamar a la lluvia (Bellenger, 1988; Rosing, 1996).

Entre los seis santuarios principales o calvarios mayores, cuatro representan a los varayuq. Esta litomorfización de las autoridades tradicionales simboliza en posición y en lugar su poder y sus áreas de influencia. Los otros dos santuarios principales restantes, son los más importantes y son considerados por los insulares como los antiguos lugares de residencia de los ancestros fundadores de la isla de Taquile (Duviols, 1978). Uno, denominado kuwanu, está situado al sur, en uray lado, la mitad baja de la comunidad, mientras que el otro se levanta en el norte en hanaq lado, la mitad alta de la comunidad en el lugar llamado Mulsina, desde donde vela por el destino del conjunto de la isla de Taquile. En este lugar sagrado se practica todos los años el Gran pago con el objetivo de reunir, mientras dura el ritual, a los principales Apu que pueblan el horizonte de este mundo lacustre.

5 Encontramos que el este como orientación principal de los chamanes durante los rituales lo es también en numerosos santuarios andinos (Girault, 1988; Martínez, 1989; Wachtel, 1973; 1990).

6 El término aymara churu designa una porción de terreno o un pedazo de tejido (Bertonio, 1984 [1612], Segunda parte: 94). Verónica Cereceda observa que el término chhuru designa «algunas franjas de tejidos de Isluga, [...] o pequeños campos rituales cerrados» (1978: 1030). 


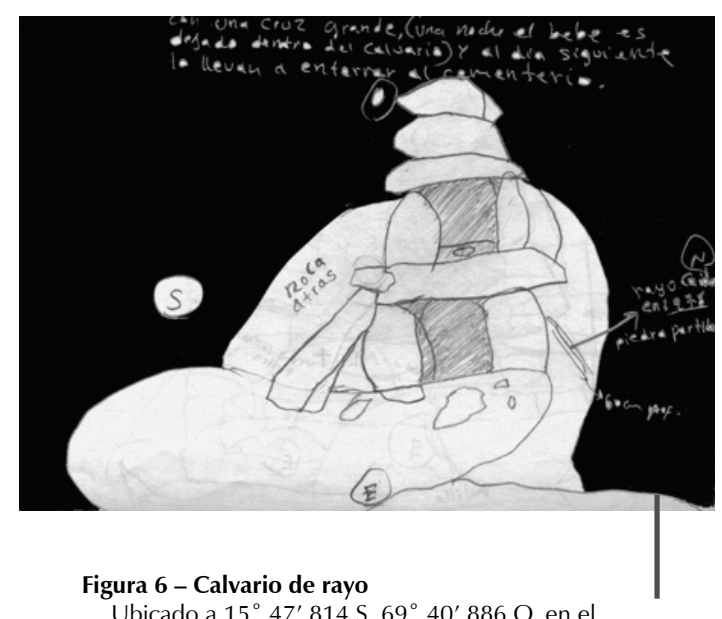

Ubicado a $15^{\circ} 47^{\prime} 814 \mathrm{~S}, 69^{\circ} 40^{\prime} 886 \mathrm{O}$, en el territorio de Kollata

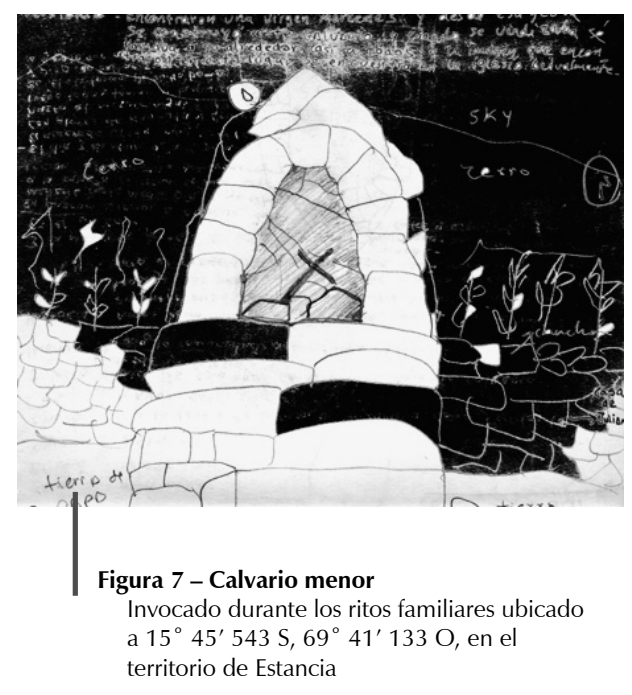

\section{MULSINA: CENTRO GEOGRÁFICO DE UN ESPACIO CIRCUMLACUSTRE SAGRADO}

En la Pascua de Resurrección se desarrolla en Mulsina, en presencia de las principales autoridades tradicionales, el gran ritual o Gran pago de la isla de Taquile. Otra ceremonia semejante pero más modesta, sincronizada con la de Mulsina, se realiza en el territorio central de Chuñopampa en el lugar denominado Taquile pata ( $15^{\circ} 45^{\prime} 919$ S, 69 41' 204 O). A esta ceremonia dirigida por un chamán asociado a uray lado, la parte baja, están invitadas las autoridades de segundo rango de la comunidad.

El santuario de Mulsina, que recibe a los protagonistas del ritual principal, se extiende por un terreno de cerca de $100 \mathrm{~m}^{2}$ rodeado por un muro de piedra. Este cerco está dividido según un eje norte/sur en dos terrenos: uno, situado al oeste, destinado a las autoridades principales y sus invitados, el otro, al este, llamado canchón sagrado, reservado al paqu que oficia y a sus secretarios o asistentes.

En medio del muro situado al este del canchón, el terreno reservado a los oficiantes, se levanta un monumento de forma cónica que representa a Mulsina, el principal Apu de Taquile.

Con ocasión del Gran pago, una puerta de acceso a este espacio, cerrado el resto del año, está despejada para permitir el acceso del chamán y de sus asistentes que llevarán a cabo el rito fuera de la vista de las autoridades y de los invitados.

Actualmente, el conjunto del ritual principal es esencialmente realizado dentro del cerco de Mulsina. Pero en otras épocas y hasta mediados del siglo XX, una parte del Gran pago de Mulsina utilizaba dieciocho receptáculos de ofrendas o churu, situados al exterior del cerco. Estos estaban dispuestos en las proximidades y en arco de círculo al sur de Mulsina. Aparecían bajo la forma de un túmulo de una altura cercana a 1,80 m, en cuyo pie se levantaba un pequeño cerco, el churu santuario. Estos lugares de ofrendas específicas destinadas a las más importantes divinidades exteriores de Taquile, ya no están más en actividad y se encuentran en parte destruidos. Sin embargo, hemos identificado formalmente sus emplazamientos. 
Cuadro 1 - Localización de los dieciocho lugares de ofrendas o churu, asociados con Mulsina, para el culto de las divinidades exteriores a la isla de Taquile

\begin{tabular}{|c|c|c|c|c|c|}
\hline Id & Nombre & Tipo & $\mathrm{S} / \mathbf{1 5 ^ { \circ }}$ & $\mathbf{O} / 69^{\circ}$ & Alt \\
\hline Ex.01 & Karus churu & Churu sanctuario & $45^{\prime} 501$ & $41^{\prime} 376$ & 4100 \\
\hline Ex.02 & $\begin{array}{l}\text { San Pedro K'api } \\
\text { churu }\end{array}$ & Churu sanctuario & $45^{\prime} 506$ & $41^{\prime} 383$ & 4098 \\
\hline Ex.03 & Asuhina churu & Churu sanctuario & $45^{\prime} 520$ & $41^{\prime} 406$ & 4098 \\
\hline Ex.04 & Kancaharani churu & Churu sanctuario & $45^{\prime} 425$ & $41^{\prime} 425$ & 4097 \\
\hline Ex.05 & Atuq yani churu & Churu sanctuario & $45^{\prime} 077$ & $41^{\prime} 289$ & 4093 \\
\hline Ex.06 & Q'illu rani churu & Churu sanctuario & $45^{\prime} 557$ & $41^{\prime} 412$ & 4090 \\
\hline Ex.07 & Tuturani churu & Churu sanctuario & $45^{\prime} 554$ & $41^{\prime} 397$ & 4090 \\
\hline Ex.08 & Winuyu churu & Churu sanctuario & $45^{\prime} 539$ & $41^{\prime} 392$ & 4088 \\
\hline Ex.09 & $\begin{array}{l}\text { Wawayuq Q'ipiq } \\
\text { churu }\end{array}$ & Churu sanctuario & $45^{\prime} 342$ & $41^{\prime} 390$ & 4087 \\
\hline Ex.10 & Pukara churu & Churu sanctuario & $45^{\prime} 512$ & $41^{\prime} 359$ & 4092 \\
\hline Ex.11 & Copacabana churu & Churu sanctuario & $45^{\prime} 510$ & $41^{\prime} 341$ & 4100 \\
\hline Ex.12 & Wak'a illa churu & Churu sanctuario & $45^{\prime} 491$ & $41^{\prime} 332$ & 4101 \\
\hline Ex.13 & Rit'i urqu churu & Churu sanctuario & $45^{\prime} 490$ & $41^{\prime} 336$ & 4102 \\
\hline Ex.14 & Illampu churu & Churu sanctuario & $45^{\prime} 485$ & $41^{\prime} 345$ & 4103 \\
\hline Ex.15 & Chuqi illa churu & Churu sanctuario & $45^{\prime} 487$ & $41^{\prime} 348$ & 4103 \\
\hline Ex.16 & Ventilla churu & Churu sanctuario & $45^{\prime} 477$ & $41^{\prime} 347$ & 4104 \\
\hline Ex.17 & Santa Taraco churu & Churu sanctuario & $45^{\prime} 475$ & $41^{\prime} 351$ & 4104 \\
\hline Ex.18 & Amantani churu & Churu sanctuario & $45^{\prime} 466$ & $41^{\prime} 360$ & 4105 \\
\hline
\end{tabular}

Para la correspondencia entre estos lugares de ofrendas situados en la isla de Taquile y la localización geográfica fuera de la isla, de las divinidades a las cuales están destinadas, véase el cuadro 2

Id: identificación del santuario y código de clasificación en nuestra base de datos (Ex. para exterior)

Nombre: nombre dado por los chamanes de Taquile a los lugares de ofrendas reservadas a las divinidades exteriores

Tipo: clasificación del tipo de santuario y de su funcionalidad

S y O: coordenadas sur y oeste levantadas con un sistema de posicionamiento global (GPS)

Alt: altura del santuario en metros

Las informaciones reunidas precedentemente, conjugadas con el profundo conocimiento de la geografía local y la localización precisa de los numerosos santuarios, nos permitirán aprehender a través de la gestualidad del chamán, los principales elementos que participan en la materialización y la animación del espacio sagrado durante el ritual.

\section{LA MATERIALIZACIÓN DEL ESPACIO SAGRADO}

Antes de poder preparar las ofrendas, el paqu, de rodillas, con la mirada dirigida hacia el este solicita permiso para poder oficiar. Para ello, primero materializa el espacio del cual él es el centro invocando según un orden preciso aquellas divinidades que fijan los límites del espacio 
que va a ser animado. Durante largo tiempo, no había logrado entender este orden, ocultado celosamente por los oficiantes, y esto por varias razones. La primera, porque el nombre de las divinidades invocadas es generalmente pronunciado en voz baja o porque el espacio que les corresponde es designado mediante un esbozo de gesto. La segunda razón porque, aún cuando se logra aislar el nombre idóneo, a menudo resulta difícil localizarlo en el espacio real porque los lugares correspondientes a veces son invisibles para alguien no iniciado y están situados en ciertos casos a más de cien kilómetros del santuario de Mulsina. Finalmente, con mucha dificultad se encontrará en un mapa los nombres de los lugares invocados, exteriores a la isla de Taquile, porque no corresponden, la mayor parte del tiempo, a la toponimia local sino que designan desde el santuario de Mulsina la dirección o el punto cercano más conocido del lugar de residencia de la divinidad. Tan sólo después de varios meses, dirigiéndonos al lugar cada vez que fue posible, pudimos completar la lista y separar el conjunto de los santuarios exteriores invocados desde la isla de Taquile durante los rituales, y establecer así la correspondencia con su nombre local.

La primera divinidad invocada desde Mulsina es Rit'i urqu, o la montaña de nieve en quechua, situada a más de doscientos kilómetros al este de Taquile, en la Cordillera Real de Bolivia, y conocida localmente bajo el nombre de Janq'u uma, en aymara: blanca agua. Esta divinidad es, sin discusión, la más importante de la región; ella determina la orientación mayor de los rituales efectuados desde Taquile, así como la de los suyu y de los campos de cultivo.

La segunda divinidad es Asuhina, una montaña que domina la ciudad de Puno. Ésta está situada al oeste de Taquile, opuesta a Rit'i urqu.

Estas dos divinidades forman un primer eje este/oeste cuyo centro se considera que es Mulsina ${ }^{7}$.

La tercera divinidad se llama Karus, domina la península de Capachica, al noroeste de Taquile. Se la considera como la más poderosa divinidad exterior, la más cercana a Taquile.

La cuarta divinidad es Copacabana, situada en Bolivia, quien con Karus constituye un segundo eje, noroeste/sureste. Este eje ritual es extremadamente importante ya que es considerado como una división del lago, un taypi —en aymara, lo que está situado en el medio-, o el eje acuático del lago (Bouysse-Cassagne, 1978).

En la antigua división del espacio sagrado, visto desde Taquile, se consideraba que las divinidades circumlacustres estaban distribuidas en dos mitades situadas de un lado y de otro de este taypi o eje noroeste/sureste. Las divinidades situadas al este de este eje, como Rit'i urqu, eran consideradas como formando parte de uray lado o la parte de abajo, que corresponde al este y a la dirección en que el sol aparece. Las divinidades situadas al oeste de este eje eran designadas como pertenecientes a Hanaq, la parte de arriba, que corresponde a la dirección del sol poniente. Hay que destacar que nuevamente

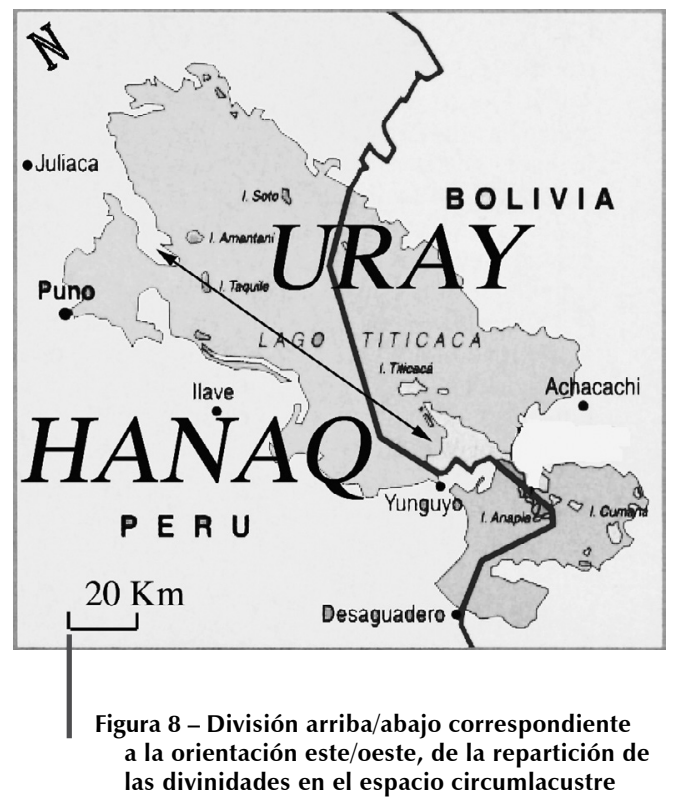

7 Aunque estas dos cumbres estén situadas a diferentes distancias de Taquile, Rit'iurqu a más de 200 km mientras que Asuhina está a menos de $40 \mathrm{~km}$, el observador instalado en Mulsina tendrá la impresión de ocupar una posición central. 


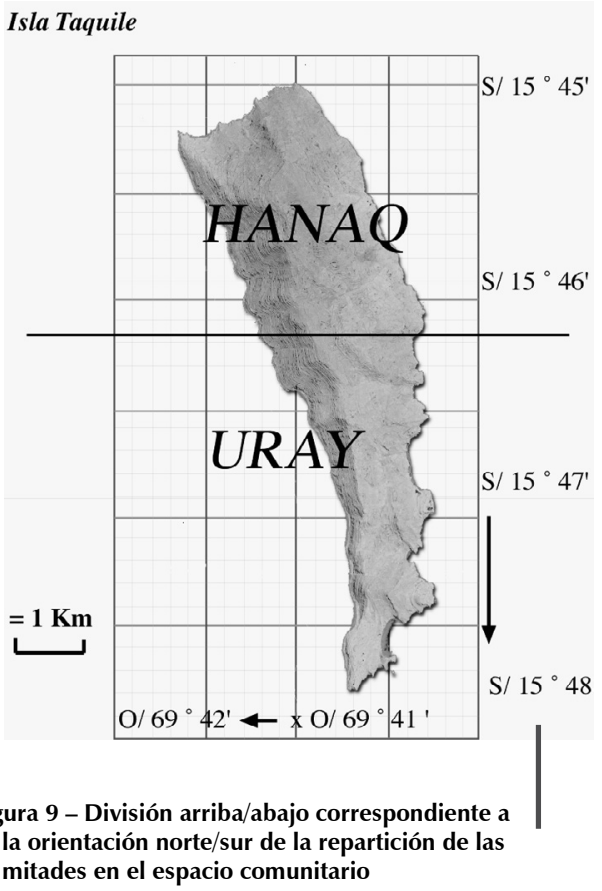

se encuentra esta división del espacio sagrado en la designación de las mitades este y oeste de la plaza central de Taquile donde se realizan la mayor parte de las danzas que acompañan al ciclo festivo calendario. Finalmente, observemos que esta división alta/baja, correspondiente a las divinidades circumlacustres situadas al oeste y al este de Taquile, es perpendicular a la división arriba/abajo que divide a las dos mitades de la comunidad: Hanaq en el norte y Uray en el sur. Posiblemente de esta manera se podría emitir otra explicación sobre el origen de la muy difundida cuatripartición del espacio comunitario andino, en lo que se refiere por una parte a una división territorial arriba/abajo o norte/sur atribuida al mundo de los vivos y por otra parte, una división territorial arriba/abajo o este/oeste asimilada al mundo de los muertos y de las divinidades.

La quinta divinidad invocada, situada al noreste, es llamada Ventilla. Nos tomó mucho tiempo localizarla, porque reside en una región en donde, vista desde Taquile, hay pocos accidentes geográficos notorios. Sin embargo, su existencia era indudable, ya que numerosos altares de los calvarios de Taquile, dirigían su «mirada» en esta dirección. Durante un recorrido con nuestro chamán informante de la isla de Taquile, quien además de su lengua materna, el quechua, habla perfectamente el aymara, pudimos explorar la zona interior de Moho, sobre la ribera este del lago Titicaca e identificar a la muy poderosa divinidad conocida localmente bajo el nombre de Pallata. Desde esta montaña se distingue muy nítidamente la isla de Taquile, que para los yatiri (chamanes en aymara) locales viene a ser una de las más importantes divinidades de la región, por su situación privilegiada en el lago Titicaca.

La sexta y última divinidad invocada, como preámbulo de la animación del espacio desde Taquile, es Winuyu. Esta montaña de colores ocre se inscribe en la prolongación sur de la península de Chucuito. Junto con Ventilla constituye el último eje ritual, noreste/suroeste, que materializa el espacio sagrado desde la cumbre de la isla de Taquile.

\section{LA ANIMACIÓN DEL ESPACIO SAGRADO}

El espacio del ritual, al estar ya fijado por los puntos de anclaje exteriores vinculados mágicamente entre sí, va a poder ser animado por el chamán. Éste, en un primer momento, llama a los principales santuarios de la isla de Taquile, luego, en un segundo momento, a las divinidades exteriores de la isla, preparándoles con sus asistentes ofrendas de hojas de coca seleccionadas y agrupadas de a tres, llamadas $k^{\prime} i n t u$. Cada $k^{\prime} i n t u$ es específicamente preparado para una divinidad. Es untado con llamp'u (grasa de llama), espolvoreado con K'uwa (una hierba aromática) y humectado con vino contenido en dos conchas. El paqu sopla ${ }^{8}$ entonces sobre el $k^{\prime}$ intu en dirección de la divinidad invocada, luego lo coloca sobre una hoja de papel blanco, la misma que se deposita sobre una unkuña (tela destinada a recibir las hojas de coca, empleada durante los rituales).

8 En este contexto, la acción del chamán que sopla sobre el k'intu podría ser asimilada a la del antiguo Camac, entidad distribuidora de fuerza vital (Taylor, 2000). 


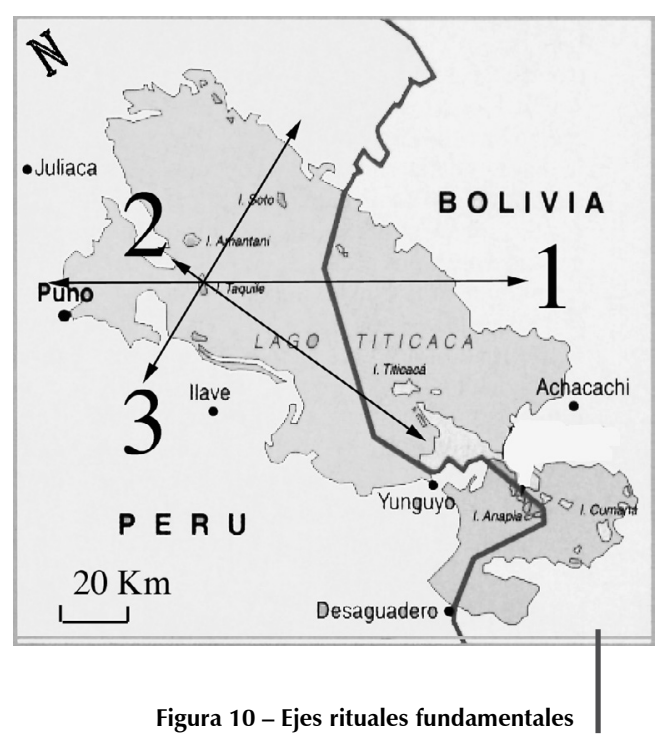

Todos estos k'intu están colocados sobre el papel según un orden preciso y su punta orientada hacia el este, en dirección de Rit'i urqu. Antes de requerir a las divinidades exteriores, en un primer momento, el paqu va a activar el espacio interior de Taquile llamando a cuatro de las principales cumbres de la isla y a dedicar a cada una de ellas un $k^{\prime}$ intu.

El primer k'intu colocado en el centro de la hoja está dedicado a Mulsina. Él es el que invita, atrae hacia sí (aysamu-) o jala a todas las otras divinidades.

El segundo k'intu colocado a la derecha del de Mulsina está dedicado a Taquile pata, segundo santuario de la isla quien acompaña a Mulsina y está bajo su protección, igual que Pukara y Kuwanu, principales cumbres o Apu de Taquile.

El tercer $k^{\prime}$ intu es preparado en honor de Pukara, la tercera divinidad de Taquile. Se lo coloca sobre la hoja de papel a la izquierda del de Mulsina.

El cuarto k'intu es el de Kuwanu, el importante santuario del territorio Kollata, al sur de la isla, antiguo lugar de residencia de uno de los dos ancestros míticos fundadores de Taquile. Es colocado debajo de la ofrenda dedicada a Mulsina.

Los dos k'intu siguientes están destinados a divinidades exteriores pero cercanas a la isla de Taquile. La primera reside en la cumbre de una montaña que domina el extremo de la península de Capachica, la segunda está escondida en los totorales en donde viven actualmente descendientes de mestizos (con aymaras) de la «gente del agua», los Urus.

El quinto k'intu es preparado para Karus, que hemos descrito como el más poderoso de los Apus exteriores, el más cercano a Taquile (véase el cuadro 2). Este $k^{\prime}$ intu es dispuesto a la derecha del de Kuwanu.

El sexto $k^{\prime}$ intu es elaborado para Kapi, una divinidad lacustre situada en la bahía de Puno (véase el cuadro 2), y se lo coloca a la izquierda del de Kuwanu.

Estas seis ofrendas constituyen el armazón central y el corazón del pago de $k^{\prime}$ intu. A partir del sétimo $k^{\prime} i n t u$, las nuevas ofrendas estarán individual y exclusivamente dedicadas a los dieciocho Apu exteriores o divinidades (de las cuales seis, ya invocadas a inicios del ritual, fijan los límites exteriores del espacio sagrado); se las colocará en espiral, en sentido inverso a las agujas del reloj, alrededor del núcleo central, comenzando de nuevo por Karus.

\section{LOS K'INTUS DE LAS DIECIOCHO DIVINIDADES CIRCUMLACUSTRES}

El primer $k^{\prime}$ intu de esta serie es colocado sobre la hoja de papel arriba y a la derecha del $k^{\prime} i n t u$ de Taquile pata. Él da inicio a una primera espiral alrededor del armazón central. Este k'intu corresponde a Karus quien domina la península de Capachica. Este Apu mayor siempre es el primer invocado de las dieciocho divinidades exteriores visibles en Taquile, desde el santuario de Mulsina.

Por otra parte, Karus corresponde, como hemos visto, al punto noroeste del eje noroeste/sureste que divide simbólicamente el lago en dos mitades, una mitad con las divinidades de uray lado, por el lado este, también designadas como divinidades del sol saliente y la otra mitad con las divinidades de hanaq lado, consideradas como pertenecientes al oeste del lago, del costado del sol poniente. 


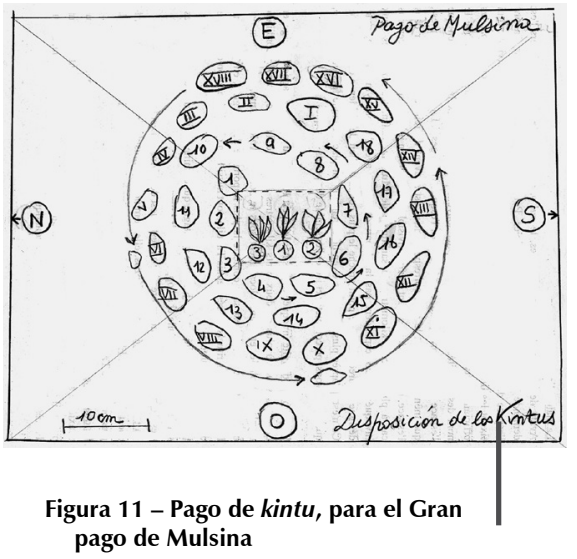

El llamado de las divinidades se efectúa en sentido inverso a las agujas del reloj y en el orden en que aparecen alrededor del lago Titicaca desde el santuario de Mulsina.

Se les invita de manera individual mediante un k'intu y se les invoca en el siguiente orden: Karus, Kapi, Asuhina, Kancharani, Atuq yani, Q'illu rani, Tuturani, Wuinuyu, Wawayuq q'ipiq urqu, Pukara, Copacabana, Wak'a illa, Rit'i Urqu, Illampu, Chuqi illa, Ventilla, Taracu, Pachatata.

A esta primera serie de ofrendas, realizada para invitar al conjunto de las divinidades invocadas, le sucede una segunda serie de dieciocho k'intu que también está dirigida a estas dieciocho divinidades exteriores para suplicarles que hagan venir abundante alimento a la isla, es decir tener buenas cosechas. Una tercera serie idéntica, siempre dispuesta en espiral, se efectúa enseguida para solicitar la protección de las autoridades

de la isla. Luego, otros tres k'intu se depositan encima del conjunto, el primero para librar a la comunidad del granizo y de las enfermedades, el segundo para el alferado (de alguna manera el padrino de la ceremonia). Finalmente, antes de colocar en el centro el último k'intu que le corresponde, el paqu hace una cruz con vino sobre el conjunto y deposita incienso en los cuatro costados de su ofrenda. Antes de doblar la hoja de papel en la que están dispuestos todos los $k^{\prime} i n t u$, se dirige a las divinidades en estos términos:

Cuadro 2 - Orden de los dieciocho k'intus iniciado por Karus y posición geográfica de las entidades divinas a las que están dedicados.

\begin{tabular}{|l|c|c|c|l|}
\hline \multicolumn{1}{|c|}{ Nombre } & Sur & Oest & Altura & \multicolumn{1}{|c|}{ Región } \\
\hline Karus (Ex. 01) & $15^{\circ} 43^{\prime} 130$ & $69^{\circ} 45^{\prime} 550$ & $4100 \mathrm{~m}$ & Península de Capachica \\
Kapi (Ex. 02) & $15^{\circ} 46^{\prime} 578$ & $69^{\circ} 54^{\prime} 296$ & $3812 \mathrm{~m}$ & En la bahía de Puno \\
Asuhina (Ex. 03) & $15^{\circ} 49^{\prime} 941$ & $70^{\circ} 02^{\prime} 830$ & $4116 \mathrm{~m}$ & Domina Puno \\
Kancharani (Ex. 04) & $15^{\circ} 52^{\prime} 827$ & $70^{\circ} 01^{\prime} 097$ & $4364 \mathrm{~m}$ & Cerca de Puno, al sur \\
Atuq yani (Ex. 05) & $15^{\circ} 54^{\prime} 620$ & $69^{\circ} 55^{\prime} 220$ & $4477 \mathrm{~m}$ & Domina Chucuito \\
Q'illu rani (Ex. 06) & $15^{\circ} 50^{\prime} 330$ & $69^{\circ} 47^{\prime} 440$ & $4000 \mathrm{~m}$ & Península de Chucuito \\
Tuturani (Ex. 07) & $16^{\circ} 05^{\prime} 133$ & $69^{\circ} 42^{\prime} 010$ & $3964 \mathrm{~m}$ & Entre Acora e Ilave \\
Wuinuyu (Ex. 08) & $15^{\circ} 58^{\prime} 870$ & $69^{\circ} 38^{\prime} 890$ & $4020 \mathrm{~m}$ & Al S-SO de la isla Escata \\
Wawayuq (Ex. 09) & $16^{\circ} 15^{\prime} 489$ & $69^{\circ} 35^{\prime} 054$ & $4627 \mathrm{~m}$ & Al sur del río Ilave \\
Pukara (Ex. 10) & $16^{\circ} 14^{\prime} 109$ & $69^{\circ} 28^{\prime} 326$ & $4342 \mathrm{~m}$ & Cerca de Juli \\
Copacabana (Ex. 11) & $16^{\circ} 09^{\prime} 983$ & $69^{\circ} 04^{\prime} 922$ & $3870 \mathrm{~m}$ & Domina Copacabana \\
Wak'a illa (Ex. 12) & $15^{\circ} 51^{\prime} 100$ & $68^{\circ} 31^{\prime} 800$ & $6150 \mathrm{~m}$ & Cordillera Real \\
Rit'i Urqu (Ex. 13) & $15^{\circ} 50^{\prime} 980$ & $68^{\circ} 32^{\prime} 000$ & $6430 \mathrm{~m}$ & Cordillera Real \\
Illampu (Ex. 14) & $15^{\circ} 48^{\prime} 800$ & $68^{\circ} 32^{\prime} 000$ & $6330 \mathrm{~m}$ & Cordillera Real \\
Chuqi illa (Ex. 15) & $15^{\circ} 43^{\prime} 272$ & $69^{\circ} 17^{\prime} 870$ & $3910 \mathrm{~m}$ & Islita (roca) del lago \\
Ventilla (Ex. 16) & $15^{\circ} 21^{\prime} 815$ & $69^{\circ} 23^{\prime} 660$ & $4430 \mathrm{~m}$ & Región de Moho \\
Taracu (Ex. 17) & $15^{\circ} 20^{\prime} 841$ & $69^{\circ} 59^{\prime} 841$ & $4208 \mathrm{~m}$ & Cerca de Taracao \\
Pachatata (Ex. 18) & $15^{\circ} 39^{\prime} 770$ & $69^{\circ} 42^{\prime} 610$ & $4115 \mathrm{~m}$ & En la isla de Amantani \\
\hline
\end{tabular}


«Ahora que ustedes, todos los Apu, están aquí, acuérdense de nosotros y protéjannos. Esta ofrenda es para Mulsina de parte de hanaq lado».

Un conjunto completo de ofrendas, similar a aquél de la mitad de arriba de la comunidad, hanaq lado, será preparado de parte de uray lado (mitad de abajo de la comunidad).

Otrora eran preparadas otras dieciocho ofrendas completas. Cada una de ellas era entregada a dieciocho peones o asistentes comisionados individualmente a los lugares de ofrendas, churu, correspondientes a las dieciocho divinidades exteriores (cf. cuadro 2). Estos churu santuarios estaban dispuestos en semicírculo al sur del santuario de Mulsina (cf. cuadro 1).

Al fin del día, cuando todo estaba listo, el paqu principal agitaba una bandera desde el cerco de Mulsina. Esta señal anunciaba el momento de incinerar

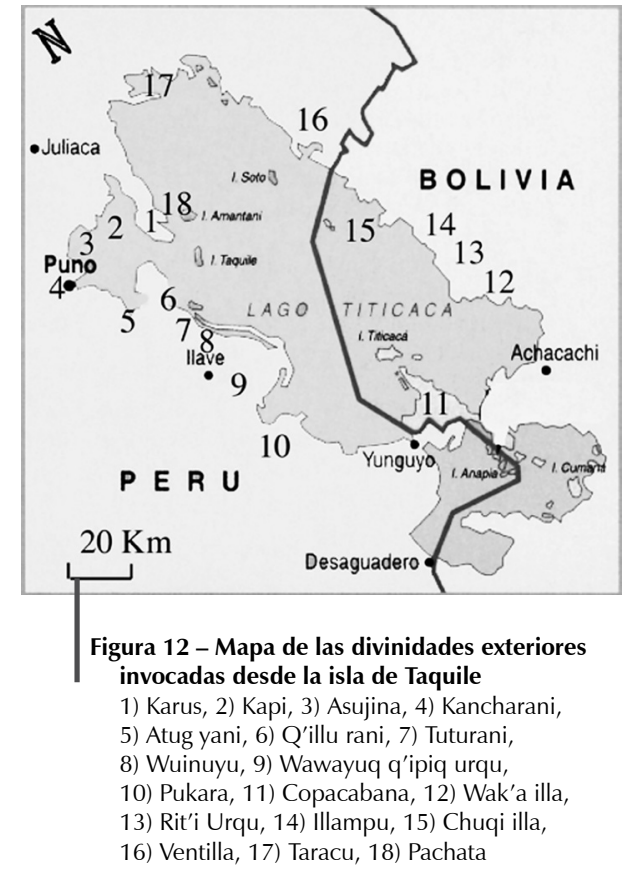

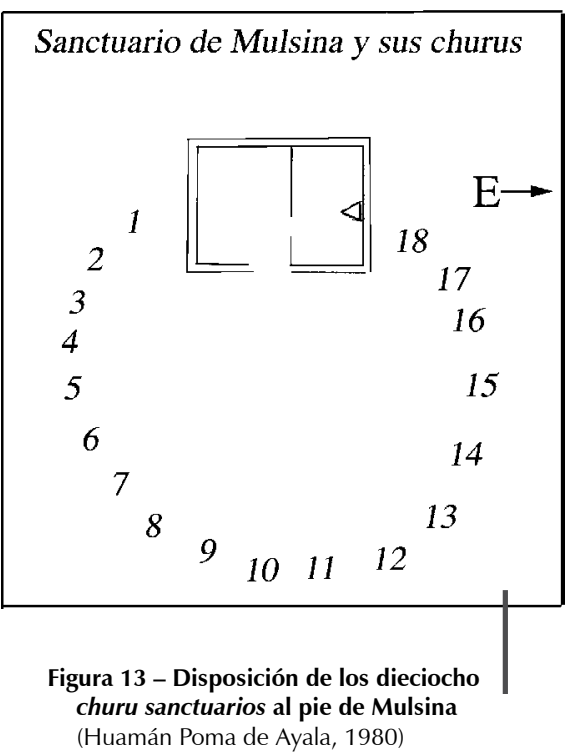

simultáneamente las ofrendas en cada una de las fogatas de los churus de las divinidades exteriores. El paqu observaba entonces la forma de las volutas de humo. Si estas eran abundantes, blancas, y subían derecho hacia el cielo, anunciaban buenos presagios y significaban que las divinidades movidas habían aceptado su alimento. En el caso en que algunas volutas correspondientes a un churu se diseminasen, el paqu preguntaba al asistente si lo había servido con convicción y le advertía acerca de las desgracias que podrían sobrevenir. Finalmente, al caer la noche, los restos de los alimentos consumidos por las divinidades eran interpretados gracias a los colores de sus cenizas: blancas para anunciar los mejores presagios, negras para predecir lo peor. El chamán principal de Taquile podía entonces, como hoy en día, comunicar confidencialmente a las autoridades los pronósticos sobre la calidad de las próximas cosechas y sobre el devenir de la comunidad.

\section{CONCLUSIÓN}

De este enfoque de la proyección de un espacio geográfico a través de la disposición de ofrendas rituales, y de la estrategia desplegada por los chamanes de Taquile para atraer hacia la isla a las fuerzas tutelares de la región, extraeremos algunos elementos de reflexión.

En primer lugar, muestra que estamos en presencia de un sistema complejo de ocupación y de manejo del espacio sagrado. Este está marcado por entidades, materializadas mediante santuarios con funciones específicas, jerárquicamente organizadas y que dominan territorios.

El chamán, operador del ritual, está siempre en el centro del espacio que va a animar. 
El lugar escogido para oficiar es determinante porque, en principio, no puede involucrar divinidades situadas fuera del campo visual ${ }^{9}$. Por esta razón, los lugares de culto más famosos están esencialmente situados en las partes altas, en zonas despejadas. Son también los lugares más peligrosos porque son propicios para concentrar fuerzas particularmente poderosas que no pueden ser recibidas sino por especialistas. Sin embargo, aunque el campo visual del chamán sea muy amplio, lo que es el caso desde el santuario de Mulsina, punto culminante de Taquile, en donde el panorama abraza prácticamente el conjunto del lago Titicaca, él no puede invocar a divinidades demasiado alejadas. Es así como algunas cumbres de la Cordillera Real, tal como el Wayna Potosí, asimiladas a poderosos Apu cercanos a La Paz, Bolivia y visibles desde la isla de Taquile, son consideradas como pertenecientes a otros espacios. Estos últimos serán animados localmente por otros chamanes cuyo centro de operación definirá los nuevos límites de su espacio ritual que involucrará entonces a otras fuerzas tutelares.

263

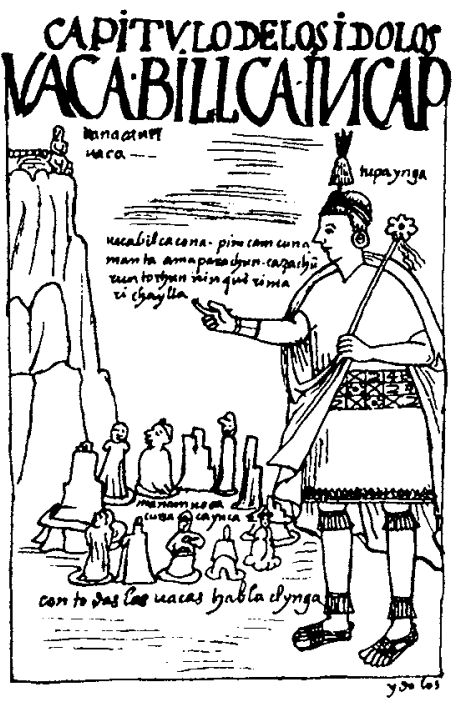

235

Figura $14-$ «Con todas las uacas habla el ynga»

(Huamán Poma de Ayala, 1980, Capítulo de los ídolos: folio 261, Tomo 1: 235)
Así, en los Andes, los espacios sagrados se montarían unos sobre otros, infinitamente, formándose o materializándose a expensas de las divinidades mayores que los presiden y de sus áreas de influencia. No pueden ser materializados y animados sino por especialistas que conocen la geografía local hasta en sus menores detalles, el nombre, el emplazamiento, la jerarquía y el orden de invocación de las fuerzas tutelares en función del lugar en el que ofician. El maestro de ceremonias también deberá saber alimentar apropiadamente a estas divinidades y en un lugar que les esté físicamente reservado. Se trata de alguna manera de una geografía de lo vivido, tal como es percibida en lo cotidiano en un lugar definido, que el chamán tendrá que saber transponer durante el ritual, con el fin de practicar su «arte de mover las montañas».

A través del Gran pago de Mulsina, vemos que el conjunto de la estrategia adoptada por los chamanes de Taquile está destinado in fine a atraer a las divinidades y a concentrar su fuerza en un solo lugar, y no a requerirlas dirigiéndoles o haciéndoles Ilegar ofrendas. Igualmente, si se observa atentamente la configuración de los diferentes santuarios que marcan el territorio de la isla ${ }^{10}$, se notará que el conjunto del dispositivo está organizado a semejanza de un sistema nervioso, cuyas ramificaciones, todas, convergen hacia el santuario de Mulsina. Durante el ritual, este santuario será igualmente regado por la energía de las entidades locales y la fuerza de las divinidades exteriores que, unidas por líneas mágicas trazadas en el espacio por el chamán, concentrarán en él su fuerza regeneradora.

9 En algunos casos específicos en el que el chamán, por razones particulares, debe oficiar de un lugar donde una divinidad invocada está escondida a causa de un accidente geográfico y, por consiguiente, fuera de su campo visual, este tendrá que recurrir a un santuario receptor intermediario. Un ejemplo de este tipo de posta especializada, denominado en Taquile calvario chaski o Apu chaski (del término quechua chaski, que quiere decir mensajero, o recibir un alimento, una bebida), está situado a $15^{\circ} 45^{\prime} 400 \mathrm{~S}, 69^{\circ} 41^{\prime} 731 \mathrm{O}$, a $3948 \mathrm{~m}$ de altura sobre el suyu de Lakayano, al norte de la isla.

10 Un estudio más amplio de todos los santuarios de la isla de Taquile se podrá consultar en un libro que será publicado proximamente por el IFEA (Bellenger, 2006). 


\section{Referencias citadas}

BELLENGER, X., 2006 (en prensa) - El espacio musical andino. Modo ritualizado de producción musical en la isla de Taquile y en la región del Lago Titicaca; Lima: Instituto Francés de Estudios Andinos.

BERTONIO, L., 1984 [ 1612 ] - Vocabulario de la lengua Aymara, 399 p.; La Paz: CERES-MUSEFIFEA . Travaux de l'Institut Français d'Études Andines, Tomo 26, edición fac-simil.

BOUYSSE-CASSAGNE, T., 1978 - L'espace aymara : urco et uma. Annales Économie Société Civilisations, 33 (5-6): 1057-1080.

BRAUNSBERGER de SOLARI, G., 1983 - Una manta de Taquile, interpretacíon de sus signos. Boletín de Lima, 29: 57-73; Lima.

CERECEDA, V., 1978 - Sémiologie des tissus andins ; les talegas d'Isluga. Annales Économie Société Civilisations, 33 (5-6): 1017-1035.

CUSIHUAMAN, A., 2001 - Diccionario Quechua, Cuzco-Collao, 211 p.; Cuzco: CBC.

DUVIOLS, P., 1978 - Un symbolisme andin du double : la lithomorphose de l'ancêtre. In: Actes

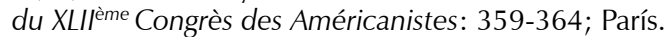

FERNÁNDEZ JUÁREZ, G., 1995 - El Banquete Aymara, Mesas y Yatiris, 570 p.; La Paz: HISBOL.

GIRAULT, L., 1988 - Rituales en las regiones andinas de Bolivia y Perú, 467 p.; La Paz: CERESMUSEF-QUIPUS.

HUAMAN POMA DE AYALA, F., 1980 - Nueva Corónica y buen Gobierno (3 tomos), 1175 p.; Lima: Ed. Siglo XXI-IEP. Edición fac-simil.

ITIER, C., 1997 - Parlons Quechua, la langue du Cuzco, 207 p.; París: I'Harmattan.

MARTÍNEZ, G., 1989 - Espacio y pensamiento (I), Andes meridionales, 206 p.; La Paz: HISBOL.

MATOS MAR, J., 1951 - La propriété dans l'île de Taquile. Travaux de I'Institut Français d'Études Andines, 3: 51-73; Lima.

ROSING, I., 1996 - Rituales para llamar la Lluvia, 574 p.; La Paz: LOS AMIGOS DEL LIBRO. Mundo Ankari 5.

TAYLOR, G., 2000 - Camac, Camay y Camasca dans le manuscrit quechua de Huarochirí. In: Camac, Camay y Camasca y otros ensayos sobre Huarochirí y Yauyos: 1-17; Cuzco: CBCIFEA.

WACHTEL, N., 1973 - Compte-rendu de Mission en Bolivie, 73 p. Informe inédito.

WACHTEL, N., 1990 - Le retour des ancêtres. Les indiens Urus de Bolivie XXème_XVème siècle : essai d'histoire régressive, 689 p.; París: Gallimard.

\section{Fuentes audiovisuales}

BELLENGER, X., 1988 - L'Appel à la pluie (Rituel quechua de l'île de Taquile) Pérou; Corto metrage La SEPT/GREM.

BELLENGER, X., 2001 - Iskay rumi taquilimanta . Video entrevista, inédito; Archivos documentales de tradiciones orales y de música; Institut Français d'Études Andines. IFEA : XBE-V2-C0033-1; Lima. 